\title{
Abordagem dietética para controle da hipertensão: reflexões sobre adesão e possíveis impactos para a saúde coletiva
}

\author{
A dietary approach to control hypertension: reflections on adherence \\ to and possible impacts on public health
}

Liliana Paula Bricarello (https://orcid.org/0000-0001-5155-0107) ${ }^{1}$

Anabelle Retondario (https://orcid.org/0000-0002-8809-5627) ${ }^{1}$

Fabiana Poltronieri (https://orcid.org/0000-0002-5075-4199) ${ }^{2}$

Amanda de Moura Souza (https://orcid.org/0000-0002-8962-798X) ${ }^{3}$

Francisco de Assis Guedes de Vasconcelos (https://orcid.org/0000-0002-6162-8067) ${ }^{1}$

\footnotetext{
${ }^{1}$ Programa de PósGraduação em Nutrição, Universidade Federal de Santa Catarina. R. Roberto Sampaio Gonzaga s/n, Trindade. 88040-900 Florianópolis SC Brasil. liliana.bricarello@ gmail.com ${ }^{2}$ Centro Universitário das Américas-FAM. São Paulo SP Brasil.

${ }^{3}$ Instituto de Estudos em Saúde Coletiva. Universidade Federal do Rio de Janeiro. Rio de Janeiro RJ Brasil.
}

\begin{abstract}
The DASH (Dietary Approach to Stop Hypertension) diet is considered a healthy eating standard and has been recommended for the control of arterial hypertension. The scope of this article was to review the scientific literature regarding the DASH diet and to verify the adherence to this food standard by the Brazilian population. An integrative review of the literature in the Medline/ PubMed, SciELO and LILACS databases was conducted in English and Portuguese. The literature on the DASH diet is extensive. However, only four studies showing adherence to the DASH diet by the Brazilian population were found. Findings from this review show that the DASH diet represents a potentially accessible and applicable intervention, which could improve the health of the population. The studies differed in the methods of evaluation. The low adherence to the diet in the Brazilian population reveals the need for nutritional actions to deal with hypertension. Innovative strategies are called for to determine how best to minimize the barriers to dissemination and greater adherence to this healthy food standard. Food plans and flexible, non-restrictive, compatible guidelines with clear objectives directed towards gradual changes with frequent monitoring by a multiprofessional health team, are suggested.
\end{abstract}

Key words DASH diet, Public health, Food consumption, Compliance, Patient adherence
Resumo A dieta DASH (Dietary Approach to Stop Hypertension) é considerada um padrão alimentar saudável, sendo preconizada para o controle da hipertensão arterial. O objetivo do artigo foi revisar a literatura sobre a dieta DASH e verificar sua adesão pela população brasileira. Realizou-se revisão integrativa nas bases Medline/ PubMed, SciELO e LILACS, nos idiomas inglês e português. A literatura sobre dieta DASH é extensa, entretanto quatro estudos mostrando a adesão pela população brasileira foram encontrados. A dieta DASH representa uma intervenção potencialmente acessível e aplicável que poderia melhorar a saúde da população. Os estudos diferiram entre si nos métodos de avaliação utilizados e a baixa adesão evidencia a necessidade de implementação de ações no âmbito da atenção nutricional ao hipertenso. Estratégias inovadoras serão necessárias para determinar a melhor forma de minimizar as barreiras para disseminação e adesão a esse padrão alimentar saudável. Sugere-se planos alimentares e orientações flexíveis, pouco restritivas, compativeis, com objetivos claros, direcionados para mudanças graduais, com monitoramento frequente de equipe multiprofissional de saúde.

Palavras-chave Dieta DASH, Saúde pública, Consumo alimentar, Conformidade, Adesão do paciente 


\section{Introdução}

A dieta DASH (Dietary Approach to Stop Hypertension), inicialmente proposta para controlar a hipertensão arterial (HA), foi concebida na década de 1990, logo após o reconhecimento de que o consumo excessivo de sódio, o alto consumo de bebidas alcoólicas e cigarro, o excesso de peso corporal e o sedentarismo estavam relacionados com aumento da pressão arterial (PA) ${ }^{1}$. Estudos sugeriram que fatores como consumo alimentar e estilo de vida dos que adotavam uma dieta vegetariana, ou ainda a associação destes, poderiam afetar a PA. Nessa perspectiva, o reconhecimento de que as dietas vegetarianas estavam associadas a níveis de PA mais baixos mostraram o importante papel do consumo alimentar de frutas, legumes, verduras (FLV), nozes e cereais integrais no manejo da $\mathrm{HA}^{1,2}$.

O propósito da criação da dieta DASH foi, então, incorporar nutrientes com potencial efeito hipotensor, provenientes de alimentos comumente consumidos pela população e não de suplementos alimentares. Basicamente, a pretensão foi recomendar alimentação que provesse os benefícios da dieta vegetariana, mas que contivesse produtos de origem animal para ser atrativa aos não vegetarianos ${ }^{1}$.

Dessa maneira, a dieta DASH preconiza o consumo de frutas, verduras, legumes, produtos lácteos com baixo teor de gordura, cereais integrais, peixes, aves e nozes e incentiva restringir o consumo de carnes vermelhas e processadas, sódio e bebidas açucaradas ${ }^{3}$.

Ensaio clínico que avaliou adultos americanos evidenciou que o consumo da dieta DASH resultou em aumento na ingestão de nutrientes que podem contribuir para redução dos níveis pressóricos, como potássio $(\mathrm{K})$, magnésio $(\mathrm{Mg})$, cálcio $(\mathrm{Ca})$ e fibras alimentares ${ }^{4}$. Por esse motivo, a DASH vem sendo recomendada para diminuição da PA em diretrizes internacionais ${ }^{5-7}$ e nacionais ${ }^{8-11}$.

Tendo em vista o potencial efeito da dieta DASH sobre a HA, esse artigo tem como objetivo revisar a literatura sobre o histórico da dieta DASH e investigar sua adesão pela população brasileira.

\section{Métodos}

Este estudo trata-se de revisão integrativa da literatura. A busca de artigos foi realizada em janeiro de 2018, sem restrição de data de publicação, nas bases de dados National Library of Medicine (PubMed), Scientific Electronic Library Online (SciELO) e Literatura Latino-Americana e do Caribe em Ciências da Saúde (LILACS), utilizando os idiomas inglês e português e os descritores, correlacionados ou isolados: Dieta DASH/ Dietary Approach to Stop Hypertension, saúde pública/public health, consumo alimentar/food consumption, conformidade/compliance, adesão do paciente/patient adhesion.

Os termos utilizados na busca estão de acordo com o Medical Subject Headings (MeSH ter$m s$ ) e/ou com os Descritores em Ciências da Saúde (DeCS), da Biblioteca Virtual em Saúde. Não foi utilizado filtro para refinar a pesquisa. Para identificar possíveis artigos elegíveis não capturados na busca, foi realizada conferência manual das listas de referências dos estudos incluídos.

Os critérios de inclusão dos artigos (elegibilidade) adotados foram: artigos disponíveis na íntegra em língua portuguesa ou inglesa que abordavam a temática dieta DASH (para fundamentar a elaboração do histórico da dieta) e a adesão à dieta pela população brasileira (para a reflexão sobre a adesão). Foram excluídos os estudos experimentais (realizados com animais).

As perguntas norteadoras da revisão foram: Quando e como foram conduzidos os estudos sobre a dieta DASH no mundo e no Brasil? Quais são os estudos que investigam a adesão dessa dieta pela população brasileira? Qual o nível de adesão da dieta DASH pela população brasileira?

Para elaboração desta revisão integrativa foram seguidos os procedimentos e etapas recomendados por Souza et al. ${ }^{12}$, conforme a descrição: 1) triagem inicial de títulos e resumos dos artigos para avaliação da elegibilidade; 2) quando as informações disponíveis nos títulos e resumos não foram suficientes, os artigos com texto completo foram lidos; 3 ) os dados foram extraídos dos artigos selecionados sobre o histórico e a adesão à dieta DASH pela população brasileira, mediante utilização de instrumento elaborado pelos autores, para simplificar, resumir e organizar os achados de modo que cada estudo foi sintetizado em uma página com informações relevantes, contendo: nome do autor, ano de publicação, tipo de estudo/tamanho amostral (n), objetivos, principais resultados e conclusões; 4) os dados foram organizados em documento do Microsoft Excel 2010 (Microsoft Corporation, Washington, EUA); e 5) foi realizada análise descritiva dos estudos selecionados, procurando estabelecer a síntese integrativa dos resultados dos distintos estudos incluídos. 


\section{Resultados e discussão}

Foram localizados 744 artigos sobre dieta DASH nas bases de dados investigadas. No entanto, após aplicação dos critérios de inclusão e exclusão, foram selecionados/incluídos 30 artigos para traçar o histórico da dieta DASH e quatro estudos que investigaram o efeito e a adesão à dieta DASH pela população brasileira (Quadro 1). O fluxograma de seleção dos artigos está apresentado na Figura 1.

\section{Histórico da Dieta DASH}

A equipe que criou a dieta DASH, patrocinada pelo National Heart Lung and Blood Institute dos Estados Unidos da América (EUA), envolveu diversos pesquisadores para propor uma modalidade de intervenção alimentar para controlar a HA. O artigo metodológico do estudo inicial foi publicado em $1995^{3}$, há mais de vinte anos.

Os primeiros achados publicados sobre a dieta DASH foram de Appel et al. ${ }^{4}$, em 1997, que realizaram ensaio clínico para testar o efeito de três padrões alimentares nos níveis de PA. O estudo distribuiu aleatoriamente 459 indivíduos adultos e saudáveis em três diferentes grupos para receberem, por oito semanas: a) dieta controle americana: rica em grãos refinados, produtos lácteos com gorduras, carnes, açúcar, poucas frutas, nozes, verduras e vegetais; b) dieta rica em Frutas e Verduras (FV); e c) dieta DASH: rica em FV, carnes magras, produtos lácteos com pouca gordura, cereais integrais, nozes e reduzida em gordura total, saturada e colesterol ${ }^{4}$. As propostas de composição nutricional das dietas utilizadas no estudo DASH são apresentadas no Quadro 2.

Os alimentos foram preparados em cozinhas dos centros de pesquisa de acordo com cardápio padrão. Os participantes almoçaram ou jantaram no local, todos os dias do estudo. Após essa refeição, os indivíduos receberam bolsas térmicas contendo alimentos para serem consumidos fora do local. Nas sextas-feiras, eles receberam refeições para o fim de semana. Os participantes foram instruídos a não beber mais de três porções de bebidas com cafeína e não mais do que duas porções de bebidas alcoólicas por dia. Dois sachês de sal, cada um com 200mg de sódio, foram fornecidos diariamente para uso. Os indivíduos registraram a ingestão de todos os alimentos, incluindo bebidas e sal. Eles indicaram se comeram alimentos fora aqueles recomendados e se não consumiram todos os alimentos do estudo. Os procedimentos para avaliar a adesão às dietas foram revisados após o primeiro grupo completar o programa. Cada centro de pesquisa forneceu reembolso e incentivos para promover a adesão ao protocolo. Testes culinários foram realizados para garantir que as dietas fossem palatáveis. A quantidade de sódio foi similar em todas as dietas ${ }^{4}$.

Como resultado, os pesquisadores encontraram que a dieta FV reduziu a pressão arterial sistólica (PAS) em 2,8mmHg ( $\mathrm{p}<0,001)$ e a pressão arterial diastólica (PAD) em 1,1mmHg ( $\mathrm{p}<$ $0,07)$, enquanto a dieta DASH reduziu a PAS em $5,5 \mathrm{mmHg}$ e a PAD em 3,0mmHg (p < 0,001), ambas em relação ao grupo controle. Considerando somente os indivíduos com HA leve ( $\mathrm{n}=$ 133), a dieta DASH foi capaz de reduzir a PAS em $11,4 \mathrm{mmHg}$ e a PAD em 5,5mmHg (p < 0,001), em relação aos controles. $\mathrm{O}$ estudo concluiu que a dieta DASH pode ser uma estratégia para reduzir e tratar a HA${ }^{4}$.

Em 2001 foi publicado, pelo mesmo grupo de pesquisadores, o estudo DASH-Sodium, que teve como objetivo avaliar se a restrição de sódio dietético teria efeito adicional sobre a redução dos níveis de PA induzida pela dieta $\mathrm{DASH}^{13}$. Esse ensaio clínico cruzado, multicêntrico, realizado nos EUA, avaliou 412 indivíduos adultos com PAS entre $120-159 \mathrm{mmHg}$ ou PAD entre $80-95 \mathrm{mmHg}$ e teve como intervenção: a) dieta rica em sódio com base na dieta americana (3450mg/dia); b) dieta intermediária em sódio $(2300 \mathrm{mg} / \mathrm{dia})$; e c) dieta com baixa ingestão de sódio (1150mg/dia). Os participantes receberam dieta típica americana, rica em: grãos refinados, produtos lácteos ricos em gorduras, carnes, açúcar, poucas frutas, nozes, verduras e vegetais por duas semanas. Após esse período, foram distribuídos de forma aleatória para receber dieta DASH: rica em FV, carnes magras, lácteos com pouca gordura, cereais integrais, nozes e reduzida em gorduras total, saturada e colesterol ou dieta americana (controle). Os dois grupos foram submetidos a cada um dos três níveis de ingestão de sódio por 30 dias. Os participantes receberam gratuitamente kits com todos os alimentos, incluindo lanches e refeições. Os resultados mostraram que a cada nível de consumo de sódio, os níveis de PA foram menores no grupo intervenção em relação ao grupo controle. $\mathrm{O}$ estudo mostrou ainda que nas duas dietas os efeitos hipotensores associados à restrição de sódio foram maiores nos indivíduos hipertensos (definido como PAS de 140 a $159 \mathrm{mmHg}$ ou PAD média de 90 a $95 \mathrm{mmHg}$, durante as três visitas de triagem) do que nos normotensos $^{13}$. 


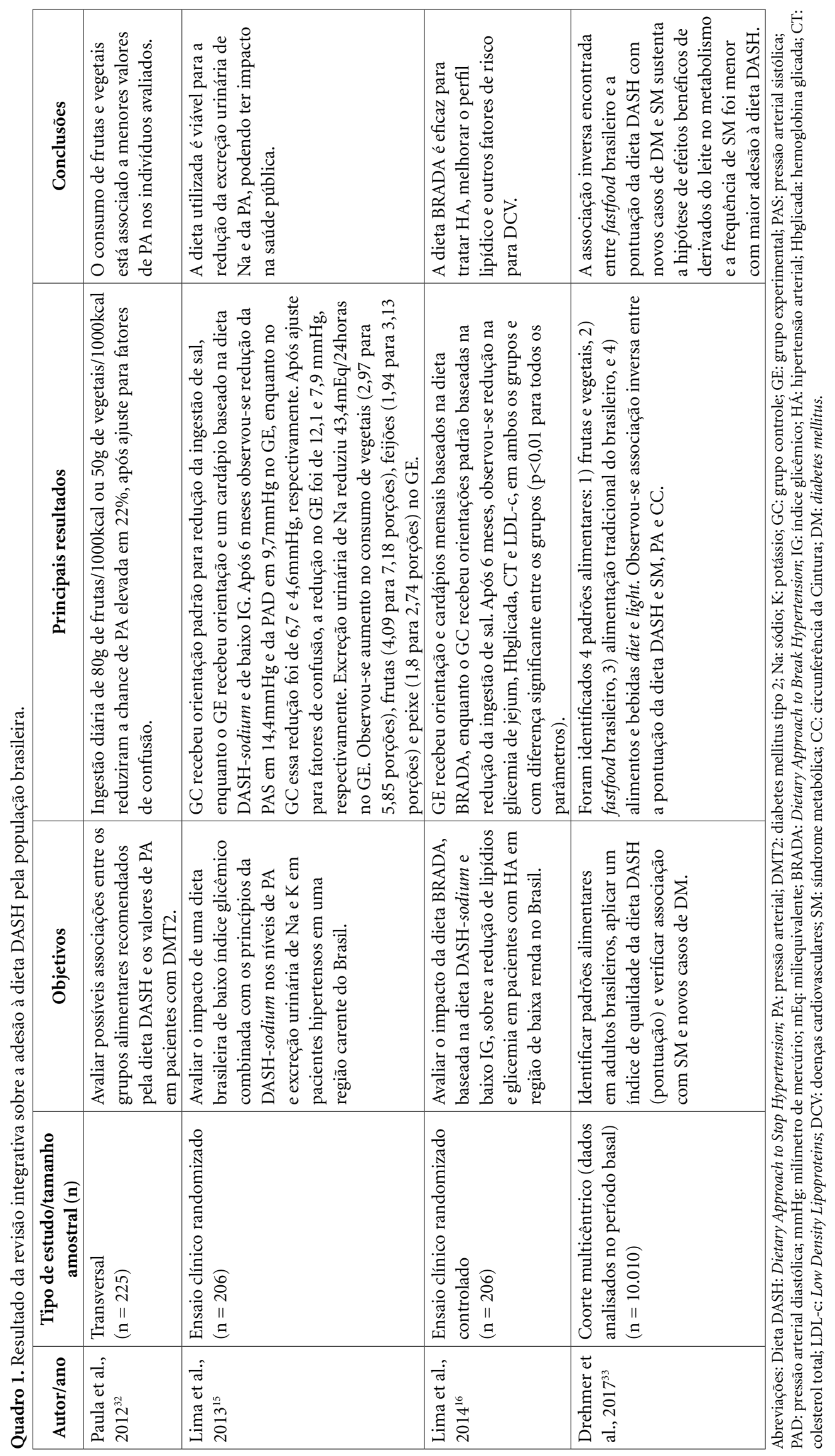




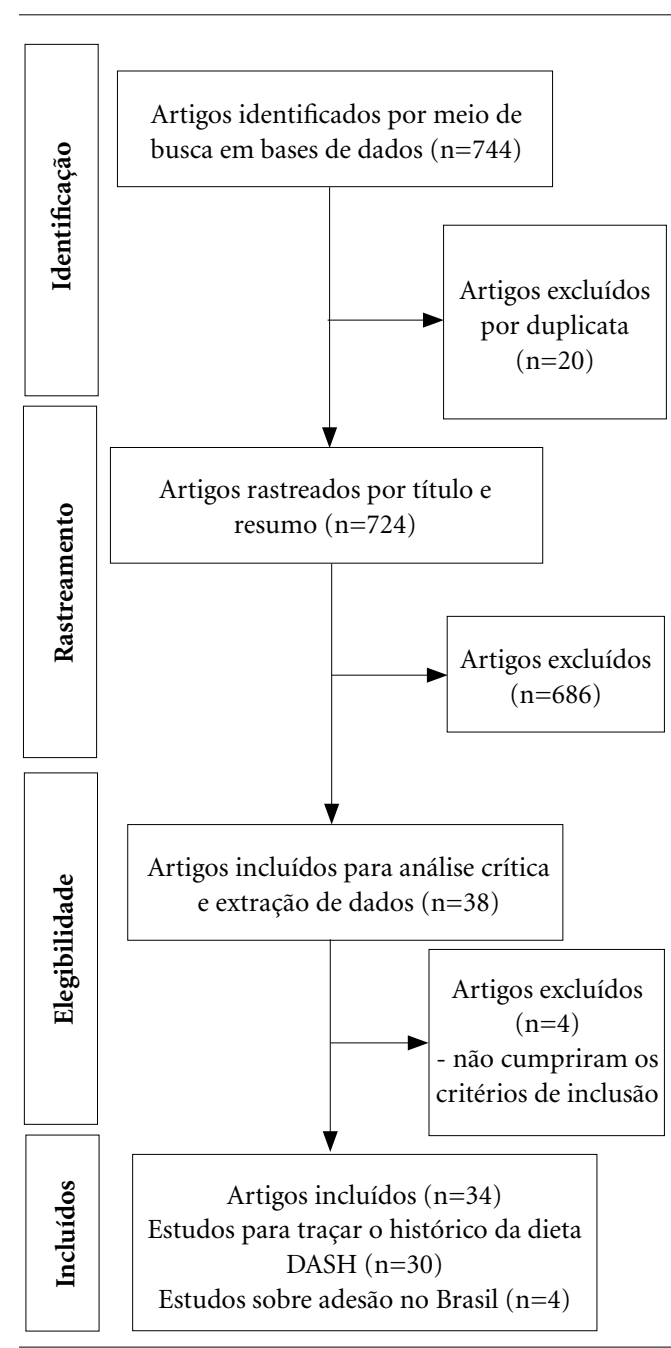

Figura 1. Fluxograma de seleção dos estudos.

Quadro 2. Composição nutricional das dietas do estudo DASH (Dietary Approach to Stop Hypertension).

\begin{tabular}{|l|c|c|r|}
\hline \multicolumn{1}{|c|}{ Nutrientes } & $\begin{array}{c}\text { Controle } \\
\text { americana }\end{array}$ & $\begin{array}{c}\text { Frutas e } \\
\text { verduras }\end{array}$ & DASH \\
\hline Gordura \% & 37 & 37 & 27 \\
\hline Saturada\% & 16 & 16 & 6 \\
\hline Monoinsaturada \% & 13 & 13 & 13 \\
\hline Poli-insaturada \% & 8 & 8 & 8 \\
\hline Carboidratos \% & 48 & 49 & 55 \\
\hline Proteínas \% & 15 & 15 & 18 \\
\hline Potássio (mg) & 1700 & 4700 & 4700 \\
\hline Magnésio (mg) & 165 & 500 & 500 \\
\hline Cálcio (mg) & 450 & 450 & 1240 \\
\hline Fibras (g) & 9 & 31 & 31 \\
\hline Colesterol (mg) & 300 & 300 & 150 \\
\hline Sódio (mg) & 3000 & 3000 & 3000 \\
\hline
\end{tabular}

Adaptado de Sacks et al. ${ }^{3}$ e Appel et al. ${ }^{4}$.
Ressalta-se que ambos os ensaios clínicos ci$\operatorname{tados}^{4,13}$ foram conduzidos sob controle rigoroso da ingestão alimentar dos participantes e seus resultados podem ser difíceis de ser reproduzidos na prática clínica, já que os pesquisadores controlaram as refeições mais do que as pessoas costumam fazer na sua rotina ${ }^{4}$.

Em 2003, foi desenvolvido o estudo multicêntrico PREMIER - Lifestyle Interventions for Blood Pressure Control ${ }^{14}$, nos EUA, desenhado para testar o efeito anti-hipertensivo de duas intervenções no estilo de vida de 810 indivíduos adultos sem HA ou com HA estágio 1 (PAS de 140 a 159mmHg ou PAD de 90 a 99mmHg). Os participantes foram distribuídos de forma aleatória em três grupos de intervenção: (1) mudança estilo de vida que implementou recomendações de alimentação saudável, aumento de atividade física, consumo controlado de sódio e álcool; (2) mudança de estilo de vida que implementou as recomendações da intervenção 1 , mais o padrão dietético DASH; ou (3) grupo controle que recebeu apenas aconselhamento nutricional. As intervenções duraram 18 meses, com 6 meses de intervenção intensiva e 12 meses de manutenção. Os participantes de ambas as intervenções (1 e 2) receberam aconselhamentos em grupo semanais durante as primeiras 8 semanas, duas vezes por semana, e mensalmente nos últimos 12 meses. Sete sessões individuais, realizadas por nutricionistas, foram intercaladas ao longo dos 18 meses. Os objetivos das intervenções foram perda de peso corporal de pelo menos $6,8 \mathrm{~kg}$ em 6 meses para aqueles com IMC $\geq 25 \mathrm{~kg} / \mathrm{m}^{2}$, pelo menos $180 \mathrm{~min} / \mathrm{semana}$ de atividade física de intensidade moderada, não mais de $100 \mathrm{mmol} /$ dia de sódio dietético e não mais de $30 \mathrm{ml} /$ dia de álcool (duas doses) para homens e $15 \mathrm{~mL} /$ dia (uma dose) para mulheres. Além disso, os indivíduos na intervenção 2 foram aconselhados a implementar o padrão dietético DASH, com as seguintes recomendações: 9-12 porções de frutas e vegetais, 2-3 porções de produtos lácteos com baixo teor de gordura e consumo de gordura total e saturada de não mais de 25 e $7 \%$ do total de calorias/dia, respectivamente. Em contraste, o grupo controle recebeu uma única sessão de aconselhamento individual de 30 minutos. Nesta sessão, os participantes receberam instruções verbais e materiais escritos que forneceram informações sobre as recomendações de alimentação saudável e o padrão dietético DASH. No entanto, nenhum aconselhamento comportamental ou contato de intervenção foi fornecido até a conclusão da coleta de dados de 6 meses $^{14}$. 
Este estudo foi o primeiro a avaliar a viabilidade da implementação da dieta DASH em indivíduos fora do ambiente de um ensaio clínico ${ }^{14}$. Após seis meses de seguimento, todos os grupos diminuíram a $\mathrm{PA}^{14}$. O controle da HA obteve melhor resultado no grupo que recebeu orientação padrão de mudança de estilo de vida associada à dieta DASH: 77\% dos indivíduos com estágio 1 de HA no período basal tiveram a PAS inferior a $140 \mathrm{mmHg}$ e a PAD inferior a $90 \mathrm{mmHg}$. No grupo que recebeu orientação padrão de mudança de estilo de vida, observaram-se resultados favoráveis em $66 \%$ dos indivíduos. Os efeitos benéficos sobre a $\mathrm{PA}$, atribuídos somente à dieta $\mathrm{DASH}$, foram inferiores aos previamente encontrados no estudo $\mathrm{DASH}^{4}$. O estudo concluiu que a dieta DASH associada a mudanças de estilo de vida diminui o risco de doenças cardiovasculares $(\mathrm{DCV})^{14}$. Porém, a redução global (mudança na PA entre o período basal e seis meses em cada grupo de tratamento menos a PA no grupo que recebeu somente orientação padrão) foi menor do que a esperada ${ }^{14}$.

A dieta DASH tem sido considerada um padrão alimentar saudável e continua sendo estudada em diversos países. Pesquisas apontam que este padrão reduz a $\mathrm{PA}^{4,15,16}$, melhora o perfil lipídico $^{17}$, contribui para controle da glicemia ${ }^{18} \mathrm{e}$ propicia menor risco de $\mathrm{DCV}^{19}$.

Nos EUA, a dieta DASH foi recomendada como parte do tratamento da HA em 1998, pelo Departamento de Saúde e Serviços Humanos, no Guia para redução da PA com $\mathrm{DASH}^{20}$ (material atualizado em 2006) ${ }^{6}$. A dieta DASH também é recomendada nas Diretrizes dietéticas para americanos nas edições de $2005^{21}, 2010^{7}$ e 2015-2020 22 e pela American Dietetic Association, American Academy of Pediatrics e American Heart Association. No entanto, alguns estudos mostraram que a dieta DASH é subutilizada entre os cidadãos dos EUA que têm HA e pré-hipertensão ${ }^{23-25}$.

$\mathrm{O}$ estudo de Mellen et al. ${ }^{23}$ avaliou hipertensos participantes do National Health and Nutrition Examination Survey (NHANES) em 19881994 e 1999-2004 e descobriu que os indivíduos com HA apresentavam escores baixos de DASH, e que a adesão à DASH foi 7,3\% menor no segundo período em comparação com NHANES 1988-1994, sugerindo que as tendências seculares minimizam o impacto da orientação dietética ${ }^{23}$.

Kim e Andrade ${ }^{24}$ usaram dados transversais do NHANES e verificaram que indivíduos com HA não pareciam seguir as diretrizes da DASH e mostrou que indivíduos com diagnóstico de HA eram menos propensos a seguir uma die- ta saudável no controle da PA do que aqueles sem diagnóstico. Em particular, indivíduos com diagnóstico de HA apresentaram ingestão significativamente maior de sódio, gordura saturada e gordura total do que aqueles que não foram previamente diagnosticados; os indivíduos obesos com HA também apresentaram adesão mais baixa à dieta $\mathrm{DASH}^{24}$. Outros estudos relataram baixos índices de adesão à DASH em indivíduos com HA e diabetes ${ }^{25-28}$.

\section{A investigação sobre a dieta DASH no Brasil}

No Brasil, a dieta DASH foi citada pela primeira vez nas "IV Diretrizes Brasileiras de Hipertensão Arterial", documento elaborado pelas Sociedade Brasileira de Cardiologia, Sociedade Brasileira de Hipertensão e Sociedade Brasileira de Nefrologia, no ano de $2004^{8}$, que recomendava dieta saudável e de baixo teor de sódio, fazendo referência ao estudo DASH-sodium ${ }^{13}$. No entanto, somente na atualização da Diretriz, em 20069 houve efetiva recomendação da dieta DASH como parte do tratamento não farmacológico da HA. Foi ressaltado que essa dieta possui grau de recomendação I e nível de evidência $\mathrm{A}$, quando existem estudos com forte recomendação na escolha e são excelentes os níveis de evidência para recomendar rotineiramente a conduta. Desde então, as atualizações de $2010^{10}$ e $2016^{11}$ da Diretriz continuam indicando a dieta DASH como parte do tratamento da HA.

Em 2012/2013, duas publicações de um ensaio clínico demonstraram viabilidade da abordagem dietética DASH e benefícios à saúde de forma consistente no Brasil ${ }^{16,17}$, onde a HA é um problema de saúde pública ${ }^{29-31}$.

Os estudos populacionais de base nacional têm apontado elevadas prevalências de HA no Brasi $^{29-31}$. Dados do Programa de Vigilância de Fatores de Risco e Proteção para Doenças Crônicas por Inquérito Telefônico (VIGITEL-2016) ${ }^{29}$ realizado pelo Ministério da Saúde (MS), indicam que a prevalência de HA autorreferida entre indivíduos com 18 anos ou mais, residentes nas capitais brasileiras, aumentou em uma década. A frequência de diagnóstico médico de HA foi de $25,7 \%$, sendo maior em mulheres $(27,5 \%)$ do que em homens $(23,6 \%)$. A pesquisa mostrou também que a PA aumenta com a idade e é maior entre os indivíduos com menor escolaridade ${ }^{29}$.

A Pesquisa Nacional de Saúde (PNS-2013) ${ }^{30}$, realizada pelo Instituto Brasileiro de Geografia e Estatística (IBGE), mediu a PA de 60.202 
adultos, das zonas urbana e rural das grandes regiões e Unidades da Federação do Brasil. Foram avaliados moradores em domicílios sorteados, utilizando aparelhos semi-automáticos digitais, calibrados. A prevalência geral de $\mathrm{PA} \geq$ $140 / 90 \mathrm{mmHg}$ foi $22,3 \%$, com predomínio entre os homens ( $25,3 \%$ vs $19,5 \%$ em mulheres), variando de $26,7 \%$ no Rio de Janeiro a $13,2 \%$ no Amazonas, com predomínio na área urbana em relação à rural $(21,7 \% \text { vs } 19,8 \%)^{30}$.

Por sua vez, dados do Estudo Longitudinal de Saúde do Adulto (ELSA-Brasil) ${ }^{31}$, que analisou 15.103 servidores públicos de seis capitais brasileiras entre 2008 e, apontaram prevalência de HA de $35,8 \%$, com predomínio entre homens $(40,1 \%$ vs $32,2 \%)^{31}$.

Dentre os fatores de estilo de vida que podem estar associados diretamente às elevadas prevalências de HA encontradas nos estudos nacionais, destaca-se a qualidade da dieta. Dados do Inquérito Nacional de Alimentação (INA 2008$2009)^{32}$, divulgada pelo IBGE, evidenciou o consumo de um padrão alimentar inadequado pela maioria dos brasileiros, caracterizado pelo alto consumo de alimentos ricos em gorduras, açúcar e sódio e pobres em micronutrientes, combinado à baixa ingestão de alimentos protetores, como frutas, verduras, legumes e grãos integrais ${ }^{32}$.

Este padrão alimentar associado a outros fatores do estilo de vida pode estar diretamente associado às elevadas prevalências de HA encontradas nos estudos nacionais ${ }^{1,29-31}$.

Conforme levantamento realizado (Quadro 1), quatro estudos sobre a dieta DASH foram desenvolvidos com a população brasileira ${ }^{16,17,33,34}$.

Um ensaio clínico foi desenvolvido por Lima et al. ${ }^{16,17}$ a fim de avaliar o impacto de uma intervenção nutricional (estilo DASH, com baixo índice glicêmico e restrição de sódio) adaptada ao padrão alimentar brasileiro, no controle dos níveis pressóricos e metabólico de pacientes hipertensos, em São Luís do Maranhão. Os autores verificaram que, após seis meses, houve redução na média da PAS em $14,4 \mathrm{mmHg}$ e na PAD de $9,7 \mathrm{mmHg}$ no grupo experimental (GE), em comparação a $6,7 \mathrm{mmHg}$ e $4,6 \mathrm{mmHg}$, respectivamente, no grupo controle (GC). Ambos os grupos (GE e GC) receberam orientação padrão para controle da HA, focada na redução da ingestão de sal. O GE, além da orientação padrão, também recebeu aconselhamento nutricional mensal fornecido por nutricionista, que se baseou nos princípios da $\mathrm{DASH}^{4}$ e nos níveis glicêmicos de alimentos. Os dados do consumo alimentar foram estimados por meio de três registros alimen- tares de 24 horas, sendo dois dias na semana e um dia no final de semana. O consumo alimentar foi modificado no GE com aumento do consumo de vegetais, passando de 2,97 para 5,85 porções; frutas (de 4,09 para 7,18); feijão (de 1,94 para 3,13) e peixes (de 1,80 para 2,74). Foram observadas modificações importantes como redução significativa de carboidratos, teor lipídico e carga glicêmica da dieta ${ }^{16,17}$.

$\mathrm{O}$ estudo mostrou a viabilidade e a eficácia de uma abordagem dietética DASH baseada no padrão alimentar brasileiro, capaz de reduzir a PA e melhorar parâmetros bioquímicos inadequados, o que pode causar grande impacto na saúde coletiva ${ }^{16,17}$. Entretanto, esses resultados foram observados em um ensaio clínico, onde os pacientes são monitorados/orientados em todas as visitas ${ }^{16,17}$.

O estudo transversal conduzido por de Paula et al..$^{33}$ avaliou adultos ( $>30$ anos) com diabetes mellitus tipo 2 a fim de identificar possíveis associações do consumo dos grupos de alimentos recomendados pela dieta DASH com valores de PA. A dieta habitual foi avaliada por registros de 3 dias com pesagem direta dos alimentos. Os pacientes receberam balanças comerciais e copos de medição. Para fins de controle, a ingestão estimada de proteína provida pela dieta foi comparada com resultados da excreção urinária de $24 \mathrm{~h}$. Os autores concluíram que o consumo dos grupos de frutas e vegetais esteve associado a menores valores de PA nos indivíduos que participaram do estudo e, por isso, seu consumo pode desempenhar papel protetor contra o aumento da PA. $\mathrm{O}$ índice de dieta DASH médio dos pacientes foi de 4,9 e nenhum dos pacientes atingiu o máximo de oito pontos no índice criado, o que significa que os pacientes cumpriram apenas cerca de 50\% das recomendações da dieta DASH $^{33}$.

O Estudo ELSA-Brasil ${ }^{34}$ avaliou indivíduos com idade entre 35 e 74 anos, em seis capitais brasileiras. Os dados do consumo alimentar foram coletados por meio de questionário de frequência alimentar (QFA). Um índice de qualidade da dieta DASH foi criado para avaliar a adesão à esta dieta. A pontuação considerou a ingestão de frutas, vegetais, nozes e legumes, grãos integrais e produtos lácteos com baixo teor de gordura, bem como de carnes vermelhas e processadas, sódio e bebidas açucaradas. Para cada um desses componentes, os indivíduos foram classificados em quintis específicos por sexo, de acordo com a ingestão, sendo os valores atribuídos a cada quintil ( 1 para a menor ingestão e 5 para maior ingestão, exceto para sódio, carnes vermelhas e 
processadas e bebidas açucaradas para as quais a pontuação foi invertida). Os dados mostraram que a frequência de síndrome metabólica foi menor com maior adesão à dieta DASH. Aqueles com maior adesão à dieta DASH eram mais frequentemente mulheres, idosos, brancos, com diploma universitário, fumavam menos, bebiam menos álcool, eram mais ativos e tinham menor índice de massa corporal (IMC) ${ }^{34}$.

Ressalta-se que os estudos na população brasileira diferiram nos métodos de avaliação utilizados para identificar a adesão com a dieta DASH, que incluiu desde abordagens subjetivas, como a avaliação da ingestão dietética para a comparação com as propostas da dieta DASH e a utilização de sistemas de pontuação DASH, até abordagens objetivas, como a medida da excreção urinária de 24 horas. Entretanto, ainda não há um consenso sobre a forma mais correta de avaliar a adesão à dieta DASH.

Percebe-se que essa adesão não foi relatada de forma detalhada em todos os estudos e a baixa adesão exige atenção. Em alguns deles, o método utilizado para avaliação da adesão não está claro, tendo em vista que esse não era o objetivo principal do estudo. Os motivos e barreiras da baixa adesão, observados em diferentes contextos clínicos, devem ser investigados para que seja possível disseminar os conceitos da dieta DASH para pacientes hipertensos.

Esses dados mostram a importância de desenvolvimento de instrumentos de avaliação da adesão à dieta DASH para a saúde coletiva e a necessidade de se preencher a lacuna em relação à adesão ao tratamento não medicamentoso. A maioria dos métodos existentes para avaliar a adesão é focada no tratamento medicamentoso, mesmo que a literatura venha apontando que há grande dificuldade de adesão ao tratamento não farmacológico ${ }^{35,36}$.

\section{Reflexões acerca da adesão ao padrão DASH}

A Organização Mundial de Saúde, em 2003, definiu adesão ao tratamento como "a medida que o comportamento de uma pessoa - em tomar a medicação, seguir a dieta prescrita e/ou implementar mudanças de estilo de vida - corresponde às recomendações fornecidas por um profissional da saúde”. Assim, o termo adesão tem sido adotado por diversos autores para definir o fenômeno, por expressar participação voluntária e ativa por parte do indivíduo no desenvolvimento e ajuste do plano de cuidados $23,24,26,35,36$.
Segundo Steinberg et al..$^{25}$, há uma série de potenciais barreiras à adesão ao padrão alimentar DASH. Essas barreiras incluem profissionais da área da saúde que não têm conhecimento na área da nutrição, pacientes que não foram orientados de forma adequada em relação aos potenciais benefícios da intervenção dietética para o tratamento da PA e os custos de uma dieta saudável para o coração, em comparação com os custos dos alimentos ultraprocessados, de alto valor calórico e pobres em nutrientes ${ }^{25}$.

A baixa adesão ao tratamento nutricional pode ocasionar ajustes na terapêutica devido à falta de resultados positivos, frustração dos profissionais de saúde e aumento de custos no cuidado à saúde com elevação das taxas de hospitalização e tratamento das complicações ${ }^{35,37}$.

$\mathrm{O}$ atual modelo econômico de saúde, que prioriza o tratamento da doença e não sua prevenção, também é uma barreira para aumentar o número de indivíduos que recebem intervenção visando mudanças no estilo de vida, com ênfase na alimentação adequada ${ }^{25}$.

Sabe-se que o aconselhamento nutricional eficaz e individualizado nem sempre está disponível à grande parte da população brasileira, tendo em vista que faltam nutricionistas e a maioria dos profissionais enfrenta uma agenda de atendimentos lotada. O sucesso do tratamento é um processo multifatorial que se estabelece por meio da parceria entre o profissional da saúde e o paciente, que envolve aspectos que vão desde o número de atendimentos até o desenvolvimento da consciência para o autocuidado e manutenção da busca pela saúde, capaz de formar atitudes, hábitos e práticas alimentares saudáveis ${ }^{38}$.

Estudo realizado no Paraná investigou a adesão aos tratamentos farmacológico e não farmacológico da HA na atenção primária e identificou fatores associados em 385 hipertensos de 20 a 79 anos. Os autores verificaram que a adesão ao tratamento farmacológico foi de $59,0 \%$, enquanto $69,1 \%$ referiram mudanças da dieta. A adesão à dieta associou-se à escolaridade inferior à $4^{\mathrm{a}}$ série e ao fato de ter recebido no mínimo uma consulta ao ano. Tais resultados evidenciam a importância de ações integrais na atenção ao hipertenso ${ }^{39}$.

No dia a dia, o caminho mais comum é a prescrição de medicação em vez de uma discussão sobre a modificação do estilo de vida. Ainda, dada a disponibilidade de medicamentos anti -hipertensivos de baixo custo, a abordagem farmacológica acaba sendo adotada em larga escala no curto prazo ${ }^{25}$. Assim, é necessário encontrar uma maneira de fazer do aconselhamento sobre 
o estilo de vida no sistema de saúde (incluindo a orientação alimentar) uma realidade para pacientes com HA e pré-hipertensão, a fim de prevenir problemas cardiovasculares e evitar a necessidade de tratamento.

O estudo de Kim e Andrade ${ }^{24}$ verificou que os indivíduos diagnosticados com HA não parecem estar envolvidos em comportamentos alimentares saudáveis e que os resultados podem ser interpretados como evidências de que esses indivíduos estão insuficientemente informados sobre a dieta DASH ou não informados em absoluto. Por outro lado, os resultados podem indicar resistência à mudança na dieta ou preferências individuais no consumo de alimentos. Portanto, a compreensão dos padrões sociais nas trajetórias das mudanças no comportamento da saúde, após um diagnóstico, pode ajudar a identificar oportunidades de intervenções que diminuam a morbidade e a mortalidade ${ }^{24}$.

Os autores chamam a atenção para o fato de que dentro do espectro do gerenciamento adequado da HA existem oportunidades para aumentar a conscientização sobre as recomendações nutricionais usando mensagens especificamente adaptadas às pessoas com HA. As mensagens podem incluir a importância de administrar sua saúde; o papel que a dieta desempenha na redução do risco de complicações de doenças crônicas; e os efeitos complementares de uma dieta saudável em combinação com a medicação anti-hipertensiva (para contrariar a tendência de escolher a medicação em detrimento a dieta saudável $)^{24}$.

Ainda, de maneira geral, um dos desafios para a saúde coletiva continua a ser a ampla disseminação e a "tradução"/adaptação da dieta DASH para a população ${ }^{25}$. Alcançar este objetivo requer a compreensão dos potenciais fatores determinantes da baixa adesão a um padrão alimentar saudável, nesse caso específico, da dieta DASH.

Vale ressaltar a multiplicidade de fatores determinantes do consumo alimentar de uma população (biológicos, econômicos, sociais, oferta/ disponibilidade dos alimentos etc. ${ }^{40}$. Entre os fatores biológicos, o sabor, que é uma característica sensorial do alimento, é apontado como um dos principais determinantes do consumo ${ }^{40}$. Nos fatores econômicos são incluídos a renda familiar, o custo dos alimentos e a escolaridade ${ }^{40}$. A oferta e a disponibilidade dos alimentos abrangem as influências do meio ambiente na aquisição dos alimentos e quanto aos determinantes sociais estão relacionados à estrutura, dinâmica e influência da família ${ }^{40}$.
Alimentos de alta densidade energética com deficiência de micronutrientes são altamente acessíveis e disponíveis em todos os ambientes. Isto é uma realidade para indivíduos de todos os níveis socioeconômicos, sendo que em áreas de menor renda os alimentos que constituem a dieta DASH podem estar menos acessíveis ${ }^{25}$. Apesar dessa barreira, uma pesquisa realizada em Boston (Massachusetts, EUA) com indivíduos de diferentes níveis socioeconômicos demonstrou que a dieta DASH pode ser adotada entre indivíduos de baixa renda ${ }^{41}$. Portanto, alterar o ambiente alimentar é uma tarefa desafiadora que exige mudanças na rotina da comunidade e das ações políticas.

Conforme o "Guia Alimentar para a população Brasileira" "42, as recomendações sobre alimentação saudável devem levar em conta o impacto das formas de produção e distribuição dos alimentos sobre a justiça social e a integridade do ambiente. Outro aspecto importante diz respeito à autonomia para escolhas alimentares mais saudáveis que dependem do indivíduo, mas também do ambiente onde ele vive. Isso inclui a forma de organização da sociedade e suas leis, os valores culturais e o acesso à educação e a serviços de saúde. Por exemplo, morar em bairros ou territórios onde há feiras e mercados que comercializam FLV facilita a adoção de um padrão alimentar mais saudável ${ }^{42}$.

Além disso, muitos profissionais da saúde se concentram apenas em orientar restrição da ingestão de sódio para controle da HA, pois muitos desconhecem o potencial da dieta $\mathrm{DASH}^{43}$. No entanto, os nutricionistas que são os profissionais habilitados legalmente para prescrever dieta, por meio de ações em educação alimentar e nutricional poderiam fazer esse aconselhamento. $\mathrm{O}$ desafio para estes é conseguir aplicar estratégias de intervenção que possam induzir a uma mudança de comportamento sustentável, que deverá levar em consideração os hábitos alimentares, os alimentos disponíveis e regionais, crenças, preocupações dos indivíduos sobre o tratamento e a cultura de cada paciente ${ }^{44,45}$.

Por outro lado, alguns pacientes não conseguem aderir à dieta por precisarem de intervenções em outras áreas de conhecimento, tais como medicina, psicologia, serviço social e educação física. Por isso, uma equipe de saúde é imprescindível, pois a adesão ao tratamento parece melhorar quando os pacientes recebem orientação da equipe multidisciplinar ${ }^{45-47}$.

Alguns determinantes facilitadores da adesão relatados na literatura encontrada foram: maior 
tempo de diagnóstico, tratamento flexível, simplificação das orientações e objetivos claros, foco em mudanças graduais, apoio social e familiar, sentimento de bem-estar, escolaridade do indivíduo, maior número de consultas ${ }^{37,45-47}$.

Uma das estratégias para melhorar a autogestão do paciente pode ser o uso de ferramentas digitais de saúde. Em 2015, o percentual de brasileiros que tinham telefone móvel celular para uso pessoal na população de 10 anos de idade ou mais era de $82,8 \%$ na área urbana e $52,8 \%$ na área rural, e o uso do telefone celular para acessar a Internet ultrapassou o uso de microcomputador nos domicílios brasileiros ${ }^{48}$.

Com a facilidade dos dispositivos móveis, essa pode ser uma oportunidade de alcançar as populações de maior risco. Alguns aplicativos e dispositivos estão disponíveis no mercado e fornecem informações que podem ser úteis para a mudança de estilo de vida e a autogestão do paciente. No Brasil, a Universidade Federal do Rio Grande do Sul (UFRGS), em parceria com o MS, desenvolveu um aplicativo gratuito para apoiar o trabalho de profissionais de saúde e pacientes que necessitam de orientação nutricional ${ }^{49}$. $\mathrm{O}$ "Dieta Dash" pode auxiliar na manutenção de uma dieta saudável e na melhor escolha de alimentos para prevenir e controlar a HA. Esse aplicativo avalia refeições com relação aos níveis de sódio, gordura saturada, colesterol e outros fatores que podem dificultar o controle da $\mathrm{HA}^{49}$. No entanto, seria importante se o aplicativo permitisse ao usuário escrever tudo o que comeu e desse ao final da refeição ou do dia uma pontuação/avaliação, pois são avaliados unicamente os alimentos.

Entretanto, alguns aplicativos não são recomendados e apresentam informações de qualidade duvidosa, sem respaldo científico. É necessário divulgar as diretrizes da dieta DASH sem alarde e com informação de qualidade.

\section{Considerações finais}

A dieta DASH representa uma intervenção potencialmente acessível e aplicável que poderia melhorar a saúde da população brasileira. Os estudos diferiram entre si nos métodos de avaliação utilizados para identificar a adesão à dieta DASH e a baixa adesão evidencia a necessidade de implementação de ações no âmbito da atenção nutricional ao hipertenso. No entanto, serão necessárias estratégias inovadoras para determinar a melhor forma de minimizar as barreiras à sua disseminação e adesão. Para melhorar a adesão à dieta DASH sugere-se planos alimentares e orientações flexíveis, pouco restritivas, compatíveis aos hábitos, cultura e ao estilo de vida da população, com objetivos claros, direcionados para mudanças graduais, com monitoramento frequente de uma equipe multiprofissional de saúde.

\section{Colaboradores}

LP Bricarello trabalhou na concepção, delineamento, pesquisa, análise e interpretação dos dados e redação do artigo. A Retondario trabalhou na análise e interpretação dos dados e redação do artigo. F Poltronieri, AM Souza e FAG Vasconcelos trabalharam na revisão crítica e aprovação da versão a ser publicada. 


\section{Referências}

1. Rouse IL, Beilin LJ, Armstrong BK, Vandongen R. Blood-pressure- lowering effect of a vegetarian diet: controlled trial in normotensive subjects. Lancet 1983; 321(1):5-10.

2. Sacks F, Kass HE. Low blood pressure in vegetarians: effects of specific food and nutrients. Am J Clin Nutr 1988; 48(3):795-800.

3. Sacks FM, Obarzanek E, Windhauser MM, Svetkey LP, Vollmer WM, McCullough M, Karanja N, Lin PH, Steele P, Proschan MA. DASH investigators. Rationale and design of the Dietary Approaches to Stop Hypertension trial (DASH). A multicenter controlled-feeding study of dietary patterns to lower blood pressure. Ann Epidemiol 1995; 5(2):108-118.

4. Appel LJ, Moore TJ, Obarzanek E, Vollmer WM, Svetkey LP, Sacks FM, Bray GA, Vogt TM, Cutler JA, Windhauser MM, Lin PH, Karanja N. A clinical trial of the effects of dietary patterns on blood pressure. DASH Collaborative Research Group. N Engl J Med 1997; 336(16):1117-1124.

5. Chobanian AV, Bakris GL, Black HR, Cushman WC, Green LA, Izzo JL Jr, Jones DW, Materson BJ, Oparil S, Wright JT Jr, Roccella EJ. Joint National Committee on Prevention, Detection, Evaluation, and Treatment of High Blood Pressure. National Heart, Lung, and Blood Institute; National High Blood Pressure Education Program Coordinating Committee. Seventh report of the joint national committee on prevention, detection, evaluation, and treatment of high blood pressure. Hypertension 2003; 42(6):1206-1252.

6. National Institutes of Health National Heart, Lung, and Blood Institute (NIH). Your guide to lowering your blood pressure with DASH, DASH eating plan. Bethesda: NIH; 2006

7. U.S. Department of Agriculture and U.S. Department of Health and Human Services. Dietary Guidelines for Americans, 2010. 7a ed. Washington: U.S. Government Printing Office; 2010.

8. Sociedade Brasileira de Cardiologia. IV Diretrizes Brasileiras de Hipertensão. Arq Bras Cardiol 2004; 82(Supl. IV):1-28.

9. Sociedade Brasileira de Cardiologia. V Diretrizes Brasileiras de Hipertensão. Arq Bras Cardiol 2006:1-48.

10. Sociedade Brasileira de Cardiologia/Sociedade Brasileira de Hipertensão/Sociedade Brasileira de Nefrologia. VI Diretrizes Brasileiras de Hipertensão. Arq Bras Cardiol 2010; 95(Supl. 1):1-51.

11. Sociedade Brasileira de Cardiologia/Sociedade Brasileira de Hipertensão/Sociedade Brasileira de Nefrologia. $7^{\text {a }}$ Diretriz Brasileira de Hipertensão Arterial. Arq Bras Cardiol 2016; 107:1-83.

12. Souza MT, Silva MD, Carvalho R. Revisão integrativa: o que é e como fazer. Einstein 2010; 8(1 Pt 1):102-106.

13. Sacks FM, Svetkey LP, Vollmer WM, Appel LJ, Bray GA, Harsha D, Obarzanek E, Conlin PR, Miller ER 3rd, Simons-Morton DG, Karanja N, Lin PH. DASHSodium Collaborative Research Group. Effects on blood pressure of reduced dietary sodium and the Dietary Approaches to Stop Hypertension (DASH) diet. N Engl J Med 2001; 344(1):3-10.
14. Svetkey LP, Harsha DW, Vollmer WM, Stevens VJ, Obarzanek E, Elmer PJ, Lin PH, Champagne C, Simons-Morton DG, Aickin M, Proschan MA, Appel LJ. Premier: a clinical trial of comprehensive lifestyle modification for blood pressure control: rationale, design and baseline characteristics. Ann Epidemiol 2003; 13(6):462-471.

15. Saneei P, Salehi-Abargouei A, Esmaillzadeh A, Azadbakht L. Influence of Dietary Approaches to Stop Hypertension (DASH) diet on blood pressure: a systematic review and meta-analysis on randomized controlled trials. Nutr Metab Cardiovasc Dis 2014; 24(12):1253-1261.

16. Lima ST, Silva NSB, França AK, Salgado Filho N, Sichieri R. Dietary approach to hypertension based on low glycaemic index and principles of DASH (Dietary Approaches to Stop Hypertension): a randomised trial in a primary care service. Br J Nutr 2013; 110(8):1472-1479.

17. Lima ST, Souza BS, França AK, Salgado JV, Salgado Filho N, Sichieri R. Reductions in glycemic and lipid profiles in hypertensive patients undergoing the Brazilian Dietary Approach to Break Hypertension: a randomized clinical trial. Nutr Res 2014; 34(8):682687.

18. Shirani F, Salehi-Abargouei A, Azadbakht L. Effects of Dietary Approaches to Stop Hypertension (DASH) diet on some risk for developing type 2 diabetes: a systematic review and meta-analysis on controlled clinical trials. Nutrition 2013; 29(7-8):939-947.

19. Salehi-Abargouei A, Maghsoudi Z, Shirani F, Azadbakht L. Effects of Dietary Approaches to Stop Hypertension (DASH) style diet on fatal or nonfatal cardiovascular diseases incidence: a systematic review and meta-analysis on observational prospective studies. Nutrition 2013; 29(4):611-618.

20. National Institutes of Health National Heart, Lung, and Blood Institute (NIH). Your guide to lowering your blood pressure with DASH, DASH eating plan. Bethesda: NIH; 1998.

21. U.S. Department of Agriculture and U.S. Department of Health and Human Services. Dietary Guidelines for Americans, 2005. $6^{\mathrm{a}}$ ed. Washington: U.S. Government Printing Office; 2005.

22. U.S. Department of Health and Human Services and U.S. Department of Agriculture. 2015-2020 Dietary Guidelines for Americans. $8^{\text {a }}$ ed. Washington: U.S. Government Printing Office; 2015.

23. Mellen PB, Gao SK, Vitolins MZ, Goff Jr DC. Deteriorating dietary habits among adults with hypertension: DASH dietary adherence. NHANES 1988-1994 and 1999-2004. Arch Intern Med 2008; 168(3):308-314.

24. Kim H, Andrade FC. Diagnostic status of hypertension on the adherence to the dietary approaches to stop hypertension (DASH) diet. Prev Med Rep 2016; 28(4):525-531

25. Steinberg D, Bennett GG, Svetkey L. The DASH diet, 20 years later. JAMA 2017; 317(15):1529-1530. 
26. Epstein DE, Sherwood A, Smith PJ, Craighead L, Caccia C, Lin PH, Babyak MA, Johnson JJ, Hinderliter A, Blumenthal JA. Determinants and consequences of adherence to the dietary approaches to stop hypertension diet in African-American and white adults with high blood pressure: results from the ENCORE trial. $J$ Acad Nutr Diet 2012; 112(11):1763-1773.

27. León-Muñoz LM, Guallar-Castillón P, Graciani A, López-García E, Mesas AE, Taboada JM, Banegas JR, Rodríguez-Artalejo F. Dietary habits of the hypertensive population of Spain: accordance with the DASH diet and the Mediterranean diet. J Hypertens 2012; 30(7):1373-1382.

28. Gao SK, Fitzpatrick AL, Psaty B, Jiang R, Post W, Cutler J, Maciejewski ML. Suboptimal nutritional intake for hypertension control in 4 ethnic groups. Arch Intern Med 2009; 169(7):702-707.

29. Brasil. Ministério da Saúde (MS). Vigitel Brasil 2016: Vigilância de fatores de risco e proteção para doenças crônicas por inquérito telefônico [documento na Internet]. Brasília: MS; 2017. [acessado 2018 Jan 18]. Disponível em: http://portalarquivos.saude.gov.br/ images/pdf/2017/junho/07/vigitel_2016_jun17.pdf

30. Andrade SSA, Stopa SR, Brito AS, Chueri PS, Szwarcwald CL, Malta DC. Prevalência de hipertensão arterial autorreferida na população brasileira: análise da Pesquisa Nacional de Saúde, 2013. Epidemiol Serv Saude 2015; 24(2):297-304.

31. Chor D, Ribeiro ALP, Carvalho MS, Duncan BB, Lotufo PA, Nobre AA, Aquino EM, Schmidt MI, Griep RH, Molina MC, Barreto SM, Passos VM, Benseñor IJ, Matos SM, Mill JG. Prevalence, awareness, treatment and influence of socioeconomic variables on control of high blood pressure: results of the ELSA-Brasil Study. PLoS One 2015; 10(6):e0127382.

32. Souza AM, Pereira RA, Yokoo EM, Levy RB, Sichieri R. Alimentos mais consumidos no Brasil: Inquérito Nacional de Alimentação 2008-2009. Rev Saude Publica 2013; 47(Supl. 1):190S-199S.

33. Paula TP, Steemburgo T, Almeida JC, Dall'Alba V, Gross JL, Azevedo MJ. The role of Dietary Approaches to Stop Hypertension (DASH) diet food groups in blood pressure in type 2 diabetes. Br J Nutr 2012; 108(1):155-162.

34. Drehmer M, Odegaard AO, Schmidt MI, Duncan BB, Cardoso LO, Matos SMA, Molina MDCB, Barreto SM, Pereira MA. Brazilian dietary patterns and the dietary approaches to stop hypertension (DASH) diet-relationship with metabolic syndrome and newly diagnosed diabetes in the ELSA-Brasil study. Diabetol Metab Syndr 2017; 13(9):13-24.

35. Rodrigues MTP, Moreira TMM, Andrade DF. Elaboração e validação de instrumento avaliador da adesão ao tratamento da hipertensão. Rev Saude Publ 2014; 48(2):232-239.

36. Figueiredo NN, Asakura L. Adesão ao tratamento anti-hipertensivo: dificuldades relatadas por indivíduos hipertensos. Acta Paul Enferm 2010; 23(6):782-787.

37. Jesus ES, Augusto MAO, Gusmão J, Mion Júnior D, Ortega K, Pierin AMG. Perfil de um grupo de hipertensos: aspectos biossociais, conhecimentos e adesão ao tratamento. Acta Paul Enferm 2008; 21(1):59-65.

38. Bueno JM, Leal FS, Saquy LPL, Santos CB, Ribeiro RPP. Educação alimentar na obesidade: adesão e resultados antropométricos. Rev Nutr 2011; 24(4):575584.
39. Girotto E, Andrade SM, Cabrera MAS, Matsuo T. Adesão ao tratamento farmacológico e não farmacológico e fatores associados na atenção primária da hipertensão arterial. Cien Saude Colet 2013; 18(6):1763-1772.

40. Estima CCP, Philippi ST, Alvarenga, MS. Fatores determinantes de consumo alimentar: por que os indivíduos comem o que comem? Rev Bras Nutri Clin 2009; 24(4):263-268.

41. Young CM, Batch BC, Svetkey LP. Effect of socioeconomic status on food availability and cost of the Dietary Approaches to Stop Hypertension (DASH) dietary pattern. J Clin Hypertens (Greenwich) 2008; 10(8):603-611.

42. Brasil. Ministério da Saúde (MS). Guia Alimentar para a População Brasileira. Brasília: MS; 2014.

43. Bell RA, Kravitz RL. Physician counseling for hypertension: what do doctors really do? Patient Educ Couns 2008; 72(1):115-121.

44. Racine E, Troyer JL, Warren-Findlow J, Mcauley WJ. The effect of medical nutrition therapy on changes in dietary knowledge and dash diet adherence in older adults with cardiovascular disease. J Nutr Health Aging 2011; 15(10):868-876.

45. Estrela KCA, Di Creddo Alves AC, Gomes TT, Isosaki M. Adesão às orientações nutricionais: uma revisão de literatura. Demetra 2017; 12(1):249-274.

46. Vivas EA, Plaza BL, Weber TK, López LB, Milla SP, Catalán AL, Gómez-Candela C. Variables predictoras de baja adherencia para el tratamiento del exceso de peso em atención primaria. Nutr Hosp 2013; 28(5):15301535.

47. Pontieri FM, Bachion MM. Crenças de pacientes diabéticos acerca da terapia nutricional e sua influência na adesão ao tratamento. Cien Saude Colet 2010; 15(1):151-160

48. Brasil. Instituto Brasileiro de Geografia e Estatística (IBGE). Ministério do Planejamento, Diretoria de Pesquisas, Coordenação de Trabalho e Rendimento. Pesquisa Nacional por Amostra de Domicílios 2015 [página na Internnet]. Rio de Janeiro: IBGE; 2016. [acessado 2017 Nov 21]. Disponível em: http://ww2. ibge.gov.br/home/estatistica/populacao/acessoainternet2015/default.shtm

49. Núcleo de Telessaúde da Universidade Federal do Rio Grande do Sul. Aplicativo dieta DASH [página na Internet]. [acessado 2017 Nov 21]. Disponível em: http://www.ufrgs.br/telessauders/desenvolvimento/ aplicativos/dietadash/

Artigo apresentado em 12/03/2018

Aprovado em 02/08/2018

Versão final apresentada em 10/08/2018 